\title{
EL AVANCE DE LA CUSTODIA COMPARTIDA EN LOS PAÍSES DEL SUR DE EUROPA. ALGUNAS CONSIDERACIONES CRÍTICAS*
}

\author{
Teresa Picontó Novales* \\ Recibido: Abril 27 de 2014 \\ Aprobado: Mayo 22 de 2014
}

\section{RESUMEN}

En los países europeos se ha generado un proceso de cambio de las familias y de las políticas familiares. Como consecuencia de ello, en los últimos años se han producido un conjunto de importantes reformas en el ámbito del derecho de familia europeo. En este artículo se analizan esas reformas y el alcance práctico de las mismas en España; pero teniendo en cuenta el marco de los países del sur de Europa (Francia, Italia y Portugal). De entrada el análisis de las mismas pondrá en evidencia un claro reforzamiento del principio de autonomía de la voluntad y el principio de co-parentalidad después del divorcio o ruptura. Finalmente se propondrán algunas reflexiones críticas desde una perspectiva de género.

Palabras claves: Derecho de familia, parentalidad, custodia compartida, derecho a la igualdad.

\section{PROGRESS OF JOINT CUSTODY IN SOUTHERN EUROPE. SOME CRITICAL APPROACHES}

\footnotetext{
*Artículo producto de investigación, derivado del Proyecto Consolider-Ingenio 2010 'El tiempo de los derechos' (CSD2008-00007).

** Profesora Titular de Filosofía del Derecho y Sociología Jurídica de la Universidad de Zaragoza. Doctora en Derecho. Correo electrónico: tpiconto@unizar.es
} 


\section{ABSTRACT}

Due to changes within families and family policies, important reforms have been implemented in the European Family Law. This article focuses on the analysis of these changes and their practical consequences in Spain, taking into account Southern Europe (France, Italy and Portugal). As a result, it is possible to perceive a reinforcement of the principles of autonomy as well as of co-parenting after divorce or breakup. The paper concludes with some critical reflections from a gender perspective.

Key words: Family Law, parenting, joint custody, right to equality

\section{O AVANCE DA CUSTODIA COMPARTIDA NOS PAISES DO SUR DA EUROPA.ALGUMAS CONSIDERAÇÕES CRÍTICAS}

\section{RESUMO}

Nos países europeus tem se gerado um processo de mudança das famílias e das políticas familiares. Como consequência disso, nos últimos anos tem-se produzido um conjunto de importantes reformas e as consequências práticas das mesmas na Espanha; mas, tendo conhecimento do marco dos países do sul da Europa (França, Itália e Portugal). Num primeiro momento a análise das mesmas colocará em evidencia um claro fortalecimento do princípio de autonomia da vontade e o princípio de coparentalidade depois do divórcio ou separação. Finalmente propor-se-á algumas reflexões criticas desde uma perspectiva do gênero.

Palavras chaves: Direito de família, parentesco, custódia compartilhada, direito à igualdade.

\section{INTRODUCCIÓN}

El proceso de cambio de las familias y de las políticas familiares que se ha producido en los países europeos a lo largo de los últimos años ha sido profundo y extenso. Como consecuencia, podemos hablar de un panorama de gran complejidad social y jurídica. En este entorno de transformaciones sociales y políticas, la ruptura conyugal y de pareja exige una amplia reorganización en el ámbito de las políticas sociales, legales y económicas de los países europeos referidas a la familia y a la protección de la infancia. Todo ello en un contexto de ajuste o redefinición de los roles masculinos y 
femeninos. Más concretamente, las relaciones entre los adultos después de la separación devienen más frágiles; pero tienen que continuar asumiendo la educación y cuidado de los hijos comunes en un ambiente difícil y, en teoría ${ }^{1}$, mucho más igualitario.

Las tensiones entre los derechos de los padres y los de las madres y de los de ambos con los derechos e intereses de los hijos, afloran con facilidad en las situaciones de ruptura familiar dando lugar a tensiones importantes entre los mismos. Las reformas legales operadas en los últimos años en el sur de Europa parecen favorecer y, en algún caso, hacen prevalecer la custodia compartida alterna. En abstracto esta tendencia iría en pro de los derechos de igualdad y libertad de los progenitores, favoreciendo la autorregulación del conflicto y la mayor implicación de los padres a la hora de asumir responsabilidades. Sin embargo, las desigualdades de género existentes y el que de facto los derechos de los adultos no siempre coinciden con el superior interés de los hijos exigen un análisis en profundidad tanto de los cambios legales operados como de la praxis judicial al respecto.

Como consecuencia de lo anterior, en los últimos años se ha producido un conjunto de reformas importantes en el ámbito del derecho de familia europeo que tienden a propiciar la equiparación de derechos de la pareja antes y después de la ruptura (Pocar \& Ronfani, 2008). En este artículo se van a revisar principalmente esas reformas y el alcance práctico de las mismas en España, pero teniendo en cuenta el marco de los países del sur de Europa (Francia, Italia y Portugal). De entrada el análisis de las mismas pondrá en evidencia un claro reforzamiento del principio de autonomía de la voluntad y el principio de co-parentalidad después del divorcio o ruptura. Esto es algo que se pone de manifiesto en relación con las potestades parentales, el ejercicio conjunto de las mismas y la guarda de hecho en todas las reformas que han tenido lugar en los derechos de familia español, francés, italiano y portugués. Ahora bien, tanto en la concreción de las reformas como en las prácticas para su implementación hay matices y problemas que exigen una consideración pormenorizada. Concretamente, estamos pensando en la definición de la guarda de hecho, dedicación cotidiana a los hijos y residencia, después del divorcio o ruptura de los padres - por convenio o acuerdo- y también cuando la medida concreta de custodia o guarda de los hijos comunes sea decidida por el juez, a falta de acuerdo entre ellos. También, se dedicará una atención específica a la custodia compartida alterna cuando no hay

1 En España concretamente, los datos existentes muestran que la praxis al respecto sigue siendo similar a la situación de etapas anteriores. Dicho de otra manera, el hombre sigue sin participar habitualmente en el ejercicio de la responsabilidad parental en términos de igualdad con respecto a la mujer. 
acuerdo de los padres, dado que esta opción va ganando progresivamente terreno como criterio judicial preferente en la implementación de las leyes de custodia compartida tras el divorcio en España en el contexto de los países del sur de Europa. Con esa finalidad se abordarán cuestiones referidas a la concreción del principio de co-parentalidad y los derechos de los hijos en las leyes y en las prácticas judiciales. Por último, se avanzarán algunas reflexiones críticas relacionadas con la existencia de circunstancias que pudieran desaconsejar la aplicación de la custodia compartida en orden a proteger el superior interés del hijo o de la hija y a cuestiones referidas a la igualdad y el principio de co-parentalidad, atisbados desde una perspectiva de género.

\section{CAMBIOS EN EL DERECHO DE FAMILIA : PARENTALIDAD Y DERECHOS DE IGUALDAD}

La tendencia de los últimos cambios legislativos ha llevado a otorgar un mayor reconocimiento del derecho a la libertad de la pareja después de la ruptura con una reducción de las exigencias legales para obtener el divorcio o la separación. Lo cual traería causa del reconocimiento del principio de la autonomía de la voluntad como un elemento determinante que rige el aspecto organizativo de la familia tanto durante la convivencia como después de la ruptura, esto es, a través de los convenios reguladores y de los pactos tras el divorcio o ruptura (Roca Trías, 2012). Por lo demás, esta tendencia iría en pro de los derechos de igualdad y libertad de los progenitores, favoreciendo la autorregulación y la mayor implicación de los padres a la hora de asumir responsabilidades con respecto a los hijos.

La separación o el divorcio de los padres requiere una completa renegociación de la dinámica familiar que permita seguir manteniendo la co-parentalidad o la parentalidad conjunta de los hijos comunes sobre la base de que las obligaciones de los padres con respecto a sus hijos continúan después de la ruptura y son exigibles tanto a los padres casados como a los que únicamente eran pareja de hecho. En este contexto, el auge del principio de co-parentalidad se apoya en la convicción de que el niño debe ser criado por sus dos progenitores incluso si se han separado o divorciado $^{2}$. En muchos de los derechos de familia europeos este

2 Como ha establecido el Tribunal Europeo de Derechos Humanos en una reiterada jurisprudencia (Sentencia Piazzi vs. Italia, $n^{\circ} 36168 / 09,2$ de febrero de 2011, Sentencia Salgueiro Da Silva Mouta vs. Portugal ${ }^{\circ}$ 3329096/96, de 21 de marzo de 2000; Sentencia Palau-Martínez, vs. Francia n ${ }^{\circ}$ 64927/01, de 16 de marzo de 2004): el principio de coparentalidad implica que la separación o ruptura de los padres no tiene incidencia sobre las reglas que rigen la responsabilidad parental. En este sentido, la limitación de los derechos de los padres debe ser excepcional. Cfr., al respecto, también, La Défenseure des Enfants 2008: 32; Ruo 2011:1017; 1051-52; Roca Trías 2012: 150-151. 
concepto de parentalidad se está traduciendo en una verdadera obligación de entenderse para los progenitores incluso si se encuentran en una situación de conflicto elevado (Ronfani, 2010). En este sentido, las leyes francesa (Ley 305/2002, de 4 de marzo) y la italiana (Ley 54/2006, de 8 de febrero), establecen una presunción fuerte en favor de la custodia compartida alterna de los hijos después del divorcio. Es decir, estos derechos de familia parecen estar disociando las reglas que se aplican a las parejas - principio de libertad o autorregulación del conflicto- de las que regulan la parentalidad o co-parentalidad -la obligación que aquí tienen los padres de entenderse-.

En definitiva, lo cierto es que progresivamente se ha ido extendiendo el discurso sobre el derecho de los hijos a sus dos progenitores y el correlativo de la necesaria implicación de los padres para el buen desarrollo de los hijos (Ruscello, 2007). Si bien, hay que subrayar ya de entrada que este discurso se ha entendido fundamentalmente en términos de los derechos de los adultos más que en el de un mayor bienestar de los hijos (Harris \& George, 2010).

Dadas las diferencias que existen en relación con las potestades parentales, el ejercicio conjunto de las mismas y la guarda de hecho entre los distintos derechos de familia (español, francés, italiano y portugués, por un lado, y los sistemas del derecho anglosajón, por otro), quizá sea oportuno hacer algunas precisiones sobre la potestad y la responsabilidad parental atribuida legalmente respecto de los hijos menores de edad y el ejercicio de la misma-custodia o guarda de hecho-. Aunque se va a atender sobre todo al derecho de familia español, las características que apuntemos se pueden hacer extensivas a los derechos francés, italiano y portugués.

Más específicamente, la potestad parental es una función integral que comprende todos los derechos y deberes de los padres en relación a sus hijos y que se refiere a las decisiones transcendentales que deben tomar los padres con respecto de sus hijos (proyecto educativo, decisiones relevantes en material de salud, religión, etc.) y la guarda de hecho comprende la atención cotidiana y las decisiones que hay que tomar en el día a día del menor, por lo que está directamente relacionada con la residencia o convivencia (Villagrasa, 2012). Así, mientras los padres viven juntos la guarda queda subsumida dentro de la potestad parental y, por ello, difícilmente se pueden diferenciar las funciones entre la potestad parental y la guarda, en tanto que titulares de la potestad parental. Cuando se produce la ruptura de la convivencia entre los 
padres hay que definir, en ese momento, el contenido de la potestad parental y de la guarda y custodia (Guilarte, 2010). Una vez rota la convivencia de los padres, es cuando se plantea si la guarda y custodia de los hijos -como parte de la potestad parental- deberá ser concedida de forma individual y exclusiva a uno sólo de los padres y un derecho de comunicación y visitas al progenitor no custodio; o, por el contrario, deberá de ser atribuida conjuntamente a ambos padres una custodia alterna -modalidad establecida y regulada por la Ley española 15/2005de acuerdo al superior interés del hijo-. En todo caso, una vez que se ha atribuido la custodia de los hijos - exclusiva o compartida-, el resto de las facultades y funciones que integran el contenido de la potestad parental corresponderá a ambos progenitores, ya que continúan compartiendo la titularidad y ejercicio de la potestad parental, a no ser que por sentencia judicial se hubiera dispuesto otra cosa, dando cumplimiento al principio de corresponsabilidad que como padres tienen (Ortuño, 2006; Castillo, 2007).

Por tanto, lo normal es que la potestad parental se conceda a ambos progenitores y que solo se prive de ella a uno de los padres en casos extremadamente graves (Picontó, 2012). De ser así, el juez tendrá que decidir en cada caso sobre la guarda y custodia compartida o exclusiva, atendiendo a como se protege mejor el interés del niño. Fijándose fundamentalmente en circunstancias como: la edad de los niños, la capacidad personal de cada uno de los padres, su concreta disponibilidad de tiempo para hacerse cargo de los hijos, la proximidad que existe entre sus respectivos domicilios, etc.

Descendiendo ya a cuestiones más concretas relacionadas con el reforzamiento del principio de co-parentalidad en las reformas legales que se están estudiando, en términos generales, se pueden distinguir tres supuestos: a) cuando haya acuerdo de los padres sobre la custodia compartida alterna de los hijos; b) que no exista tal acuerdo de los progenitores en ese sentido y que el juez adopte la medida de custodia compartida alterna cuando lo haya solicitado uno solo de los progenitores; c) que no haya acuerdo de los padres y el juez adopte la medida de custodia compartida alterna cuando no lo haya solicitado ninguno de los dos progenitores. En el primero de los supuestos, acuerdo de los padres, obviamente no se plantean problemas, si bien el juez tiene que homologar dicho acuerdo siempre que no sea contrario al superior interés del menor. Pero en los otros dos supuestos se plantean conflictos que tienen que ver con los derechos de los padres y con el superior interés de los hijos. En este trabajo voy a dedicar una especial 
atención a los supuestos en los que los padres no hayan llegado a un acuerdo, aspecto del que me ocuparé centralmente en el próximo apartado.

\section{LAS ÚLTIMAS REFORMAS LEGALES SOBRE LA CUSTODIA COMPARTIDA EN ELSUR DE EUROPA}

En los países del Sur de Europa no existe un único y estricto modelo de la custodia compartida alterna o no está reconocido de igual manera por las diversas leyes de estos países. En Italia (Ley 54/2006, de 8 de febrero) y Francia (Ley 305/2002, de 4 de marzo) se ha optado por establecer una valoración judicial prioritaria del cuidado compartido de los hijos. Concretamente, el derecho italiano establece que el juez debe valorar prioritariamente la posibilidad de que los hijos menores permanezcan bajo el cuidado de ambos progenitores, dejando la custodia exclusiva como un remedio residual y estableciendo expresamente la permanencia de la responsabilidad, de la titularidad y del ejercicio conjunto de ambos progenitores (Ruscello, 2007). Concretamente, el juez debe hacer todo lo posible porque los padres mantengan una relación y contacto continuado y así poder cumplir con sus deberes parentales.

En el caso del derecho francés, el juez puede llegar a imponer la résidence alternée aun cuando ninguno de los progenitores esté de acuerdo. Concretamente, el juez puede decidir la "residence alternée" -custodia compartida alterna- incluso en el caso de falta de acuerdo de los padres (La Défenseure des Enfants, 2008). Pero sólo con carácter temporal y a título provisional, con el objetivo de valorar su funcionalidad (Lathrop, 2008). Lo cierto es que el juez tiene mucha flexibilidad para poder adaptar las decisiones a la situación del caso: puede pedir a los padres que formulen propuestas a la vista de un acuerdo; puede establecer provisionalmente ciertas medidas hasta la decisión definitiva, etc. (La Défenseure des Enfants, 2008). Por tanto, la primera alternativa que se ofrece a los padres y al juez por la ley -art. 373-2-9 código civil francés y art. 155.2 cód. civil italiano- es la de una custodia compartida, fijada alternativamente en cada uno de los domicilios de los padres. Esta elección puede ser producto del acuerdo de los padres. Pero la ley permite igualmente al juez decidir esta modalidad de custodia compartida incluso en el caso de desacuerdo de los padres. En Francia, esta práctica se produce en 1/4 de las situaciones (La Défenseure des Enfants, 2008). En tal caso, los jueces para aplicar esta medida se basan en los informes precisos de los expertos sociales sobre la situación familiar, etc. 
Por su parte, el derecho de familia portugués ha sido el último en incorporar un sistema preferente por la guarda y custodia conjunta - guarda alternada- de los hijos tras el divorcio en los casos en que no haya acuerdo de los padres (Duarte, 2009). Concretamente, ha sido en 2008 -Ley 61/2008, de 31 de octubre-. A partir de 2008 la regla aplicable es la misma que la que rige durante la convivencia: el ejercicio conjunto de las responsabilidades parentales por los padres con independencia de si han conseguido o no un acuerdo en este sentido. Ahora bien, durante la vida en común de los padres cualquiera de los dos progenitores puede tomar decisiones sobre la vida corriente del menor. Después de la ruptura tales decisiones sobre la vida del niño corresponden al "padre que tiene la residencia o custodia del hijo" -art. 1906.3 del código civil portugués-. Esta ley de 2008 supone, entre otras cosas, un esfuerzo del legislador portugués de hacerse eco de los Principles of European Family Law regarding Parental Responsabilities, que es recomendación de la Comisión del Derecho de Familia Europeo que fueron publicados en 2007 (Boele-Woelki et al., 2007:; Duarte, 2012). El derecho de familia portugués en materia de divorcio y custodia de hijos fue modificado precisamente a raíz de las recomendaciones de esta comisión europea de expertos. Concretamente, el legislador portugués cambió el término de "poder parental" por el de "responsabilidad parental" (art. 3, Ley 61/2008). Asimismo se hizo eco del principio 320.2 de estas recomendaciones en el sentido de que a falta de acuerdo de los padres, la autoridad competente -el juez- podrá establecer la guarda conjunta de ambos salvo que entienda que esta medida es contraria al interés del hijo. Un criterio importante para los jueces a la hora de determinar la residencia del hijo es el de la disponibilidad manifestada por cada uno de los padres con respecto a que el hijo de ambos pueda tener unas relaciones habituales con el otro progenitor (Araújo, 2009).

Volviendo la atención sobre el caso español, como ya se ha apuntado, la custodia compartida alterna cuando no existe acuerdo de los padres sigue siendo muy discutida en España. De entrada, hay incluso una divergencia importante entre la ley del Estado (Ley estatal 15/2005) ${ }^{3}$ y las hasta este momento cuatro leyes de las Comunidades Autonómas (Cataluña, Aragón, Valencia y Navarra). Algunas de estas leyes, establecen la custodia compartida como preferente cuando no exista acuerdo de los padres. Por su parte, la Ley estatal 15/2005 establece la posibilidad de

3 En la actualidad se está tramitando un Anteproyecto de Ley estatal sobre el ejercicio de la corresponsabilidad parental en caso de nulidad, separación y divorcio, aprobado por el Consejo de Ministros español el 19 de julio de 2013. En su preámbulo, al final del expositivo II, los redactores del Anteproyecto afirman haberse inspirado en las leyes de custodia compartida de las cuatro Comunidades Autónomas: Aragón, Cataluña, Navarra y Valencia. 
que excepcionalmente cuando uno sólo de los padres lo solicite el juez podrá acordar, en la separación o divorcio contencioso, la custodia compartida alterna siempre que con ello se proteja el interés del menor (art. 92.8 Cód. civil). En el caso del derecho aragonés se perfila sin matices una doble vía para acceder a la custodia compartida alterna de los hijos: a) cuando exista acuerdo de los padres; b) por decisión judicial a pesar de que no exista acuerdo de los progenitores e incluso a pesar de que ninguno de ellos lo haya solicitado al juez.

También la implementación de esta medida de custodia compartida alterna en España presenta divergencias en el contexto judicial español. Si bien, a partir de 2009 la línea jurisprudencial del Tribunal Supremo (en adelante, TS) se ha ido decantando a favor de la custodia compartida alterna aunque no exista acuerdo entre los progenitores (STS. 623/2009, de 8 de octubre; STS. 94/2010, de 11 de marzo; STS. 579/2011, de 22 de julio; STS. 641/2011, de 27 de septiembre; STS. $323 / 2012$, de 25 de mayo); y ha ido recogiendo en su jurisprudencia los criterios concretos que garanticen en cualquier caso el superior interés del menor. Por otro lado, esta jurisprudencia está siendo seguida progresivamente por los jueces de familia, de primera y segunda instancia sobre todo en Cataluña y Aragón (sentencia de la Audiencia Provincial de Barcelona de 20 de febrero de 2007; sentencia de 13 de julio de 2011, sentencia del 15 de diciembre de 2011 y sentencia del 28 de septiembre de 2012 del Tribunal Superior de Justicia de Aragón). Por su parte, el Tribunal Constitucional español (en adelante TC), en su Sentencia de 17 de octubre de 2012, ponente Encarna Roca Trías, refiriéndose a la Ley estatal 15/2005, ha señalado: "Una custodia compartida impuesta judicialmente debe ser excepcional conforme a la normativa vigente (...) porque debe obligarse a los progenitores a ejercerla conjuntamente sólo cuando quede demostrado que es beneficiosa para el menor" $\left(\mathrm{FJ} 5^{\circ}\right)^{4}$. Criterio este, según el cual "el sistema de custodia compartida debe considerarse normal y no excepcional", que ha sido recogido ya por la jurisprudencia del Tribunal Supremo (STS 758/2013 de 25 noviembre).

\footnotetext{
4 En el mismo sentido, se precisa en la misma Sentencia del TC 17 de octubre de 2012 que "el interés superior del niño opera como contrapeso de los derechos de cada progenitor y obliga a la autoridad judicial a valorar tanto la necesidad como la proporcionalidad de la medida reguladora de su guarda y custodia. Cuando el ejercicio de alguno de los derechos inherentes a los progenitores afecta al desenvolvimiento de sus relaciones filiales y puede repercutir de un modo negativo en el desarrollo de la personalidad del hijo menor, el interés de los progenitores no resulta nunca preferente. Y de conformidad con este principio el art. 92 del Cód. civil regula las relaciones paterno-filiales en situación de conflictividad matrimonial, con base en 2 principios: a) el mantenimiento de las obligaciones de los padres para con sus hijos y b) el beneficio e interés de los hijos, de forma que la decisión del Juez sobre su guarda debe tomarse tras valorar las circunstancias que concurren en los progenitores, buscando siempre lo que estime mejor para aquellos" $\left(\mathrm{Fj}^{\circ} 4\right)$.
} 
En definitiva, los padres van a tener libertad para determinar las cuestiones relativas al ejercicio conjunto de la responsabilidad parental. Ahora bien, en tal situación se considera primordial proteger y garantizar los derechos y el superior interés de los hijos en las situaciones de ruptura o divorcio de los padres. Razón por la cual, el juez va a ser el garante último de la protección del superior interés de los hijos -Francia e ItaliaY en el caso español y portugués esa función va a cumplirla además del juez, el Ministerio Fiscal ${ }^{5}$. De forma que cuando exista acuerdo de los padres sobre el ejercicio conjunto de la responsabilidad parental deberá ser ratificado u homologado por el juez. El juez atenderá para su homologación a si dicho acuerdo preserva el interés superior de los hijos. Así, en la casuística de las decisiones judiciales, los jueces rechazan la custodia compartida alterna por acuerdo de los padres: a) cuando hay malas relaciones entre los padres; b) si hay alejamiento de la residencia de los padres; c) según que edad tengan los hijos; d) según cuáles sean las condiciones materiales de la residencia de uno de ellos.

Por otro lado, la existencia de determinadas circunstancias puede exigir que, en interés del niño o de la niña, el ejercicio de la responsabilidad parental solo sea concedido a uno de los padres por parte del juez, a través de una medida de custodia exclusiva. Concretamente, se trata de situaciones en las que uno de los progenitores ha tenido un comportamiento negligente, inadaptado, maltratador pero también puede ser por causa de la ineptitud de un progenitor con respecto a los derechos del otro, o por causa de un conflicto parental muy intenso, etc. Por tanto, aunque la custodia compartida constituye la regla y la custodia exclusiva la excepción, al menos en Italia y Francia (Ruscello, 2007). Y además en España, tal y como ha indicado el Tribunal Supremo, la medida de guarda y custodia compartida de los hijos no está pensada para resolver la igualdad de los padres, sino el interés de los niños (STS. 641/2011, de 27 de septiembre). Por esta razón, los Tribunales españoles acuerdan la atribución a la madre de las responsabilidades parentales "por ser más beneficioso para los menores que la custodia compartida" (Vid. Sentencia Audiencia Provincial de Madrid (Sección 22ª) 624/2013 de 29 julio).

5 Para el caso portugués, puede verse Gomes 2009: 80 y 84. Sobre la necesidad de un informe favorable del Ministerio Fiscal para que el juez pudiera adoptar la medida de la custodia compartida alterna en el caso de no existir acuerdo de los padres recogido en el artículo 92.8 del Código civil español, se ha pronunciado recientemente el Tribunal Constitucional declarando inconstitucional la exigencia de un informe favorable del Fiscal para que el juez pueda acordar la custodia compartida. Así, establece que "el interés prevalente de los hijos menores así como la inexistencia de un acuerdo entre los progenitores son motivos con suficiente peso... como para afirmar que el informe del $\mathrm{M}^{\mathrm{o}}$ Fiscal, sea o no sea favorable, no puede limitar la plena potestad jurisdiccional...". Por tanto, sólo al órgano judicial le corresponde la facultad de resolver el conflicto que se le plantea, pues exclusivamente él tiene encomendada constitucionalmente la función jurisdiccional", vid. STC 185/2012, de 17 de octubre, FJ'. 8 . 


\section{CONSIDERACIONES CRÍTICAS}

En España, como en los otros países de su entorno, la medida de custodia compartida está teniendo una gran vis attrativa. Sin embargo, una cierta distancia crítica puede ser muy oportuna. En una sociedad en la que las relaciones de poder entre los miembros de la pareja y el reparto de las tareas domésticas y las responsabilidades familiares se distribuyen asimétricamente, la aplicación de la custodia compartida no va a estar exenta de tensiones y de contradicciones.

En la sociedad española la transición de una familia de roles diferenciados por géneros a una familia igualitaria apenas si está comenzando a desarrollarse. Por ello, hay una disonancia significativa entre las orientaciones de las reformas legislativas que se están produciendo y las prácticas reales. Es cierto que en abstracto existen actitudes favorables hacia un modelo familiar igualitario, pero no son garantía de que exista una corresponsabilización parental en la realidad cotidiana. Imponer una medida como la custodia compartida alterna a falta de acuerdo de los padres - bien como criterio legal preferente o mediante su aplicación judicial- puede incluso añadir dificultades en aquellas parejas que antes de la crisis no venían compartiendo las tareas del cuidado de los hijos.

Por otro lado, el concepto de "parentalidad" puede neutralizar las relaciones de género (Bastard, 2006). Concretamente, este concepto se puede utilizar tanto para valorar las prestaciones de los padres-varonescomo para enmascarar que, a pesar de los avances y aspiraciones de la nuevas masculinidades y paternidades, los cambios en el comportamiento de los hombres en las tareas del cuidado de los hijos están todavía muy retrasados en lo referente a la noción de igualdad que está implícita en el concepto de parentalidad. Lo que explica, en parte, que muchas veces los procesos de ruptura o divorcio pongan al descubierto la desigualdad que persiste en el ámbito familiar entre las prestaciones de hombres y mujeres en el cuidado y atención de los hijos. Dicho de otra manera, si bien es cierto que en abstracto existen actitudes favorables hacia un modelo familiar igualitario, esto no garantiza que exista o vaya a existir sin más una corresponsabilización parental en la realidad cotidiana. Más concretamente, teniendo en cuenta la realidad social del reparto de roles familiares en las sociedades del sur de Europa $\mathrm{y}$, en particular la española; las denominadas leyes de igualdad o paritarias no garantizan por sí mismas la igualdad de responsabilidades. De forma que establecer una medida como la de custodia compartida si 
no hay acuerdo de los padres -bien judicialmente o como criterio legal preferente- cuando no se corresponde con el estilo de vida y la división del trabajo de muchas familias en nuestro contexto cultural puede ser arriesgado y aumentar el conflicto en aquellas parejas que antes de la ruptura no venían compartiendo las tareas del cuidado de los hijos, amén de que pueda dar lugar a desequilibrios en el poder de negociación entre ellos en el momento de la ruptura (Picontó, 2012).

La custodia compartida de los hijos, como ya se ha apuntado, requiere acuerdo, respeto y colaboración entre los padres, por lo que no debiera ser impuesta por los jueces. Además en aquellos supuestos en que la custodia conjunta no responde a la elección o acuerdo de ambos padres, su imposición supone probablemente dar a uno de ellos más de lo que quiere y al otro menos. Pero sobre todo, habrá de tenerse en cuenta que las necesidades de los niños y niñas deben ser priorizadas, lo cual exige que los acuerdos a los que haya de llegar deberán ser flexibles y garantizar que los hijos tengan el calor y el cariño incondicional de los dos hogares. Todo ello exige un esfuerzo para desarrollar criterios claros y contrastados a la hora de implementar las Leyes de la custodia compartida alterna.

\section{REFERENCIAS BIBLIOGRÁFICAS}

ARAÚJO DIAS, C. M. (2009). Una análise do novo regime jurídico do divórcio, Coimbra: Almedina.

BASTARD, B. (2006). Une nouvelle police de la parentalité?'. Enfances, Familles, Générations, 5, 11-21.

BOELE-WOELKI, K. et al. (2007). Principles of European Family Law Regarding Parental Responsabilities, Oxford, Intersentia.

CASTILLO MARTÍNEZ, C. C. (2007). La determinación en la guarda y custodia de los menores en los supuestos de crisis matrimonial o convivencial de sus progenitores. Especial consideración de la guarda y custodia compartida tras la Ley 15/2005, de 8 de julio. Actualidad Civil 2,(15), 1783-1755.

DEFENSEURE DES ENFANTS, LA. (2008), Rapport: Enfants au Coeur des séparations parentales conflictuelles. Plaidoyer pour mieux préserver l'intérêt des enfants. La Défenseure des Enfants. Recuperado de www.ladocumentationfrancaise. fr/var/storage/rapportspublics/084000714/0000.pdf 
DUARTE PINHEIRO, C. (2012). O Direito da Família Contemporâneo, $3^{\text {a }}$ Ediçao, Lisboa: AAFDL.

GOMES, A. S. (2009). Responsabilidades Parentais (De acordo com a Lei ${ }^{\circ}$ 61/2008), Lisboa: Quid Juris.

GUILARTE MARTÍN-CALERO, C. (2010). Criterios de atribución de la custodia compartida. Rev. InDret 3(4). Recuperado de www.indret.com

HARRIS, P. y GEORGE, R. (2010). Parental responsibility and shared residence orders: parliamentary intentions and judicial interpretations. Child and Family Law Quarterly 22, 151-171.

LATHROP GÓMEZ, F. (2008). Custodia compartida de los hijos, Madrid: La Ley.

ORTUÑO MUÑOZ, P. (2006). El nuevo régimen jurídico de la crisis matrimonial, Madrid: Civitas.

PICONTÓ NOVALES, T. (Ed). (2012). La custodia compartida a debate, Madrid, Dykinson-Instituto de Derechos Humanos Bartolomé de las Casas. Madrid: Universidad Carlos III de Madrid.

PICONTÓ NOVALES, T. (2012). 'The equality rights of parents and the protection of the best interest of the child after partnership breakdown in Spain', in International Journal of Law, Policy and the Family 26 (3), 378-400.

POCAR, V. y RONFANI P. (2008). La famiglia e il diritto, nueva edizione riveduta e ampliata, Bari: Editori Laterza.

ROCATRÍAS, E. (2012). Libertady Familia, Madrid: Tirant lo Blanc.

RONFANI, P. (2010). Alcune riflessioni sulla responsabilità genitoriale. Enunciati del diritto. Rappresentazioni normative e pratiche sociali. Sociologia del Diritto 1, 7-37.

RUO, M. G. (2011). Tutela dei figli e procedimenti relativi alla crisi della copia genitoriale nella giurisprudenza Della Corte Europea dei Diritti Dell'Uomo. Il Diritto di Famiglia e delle Persone, 2, 1004-1052. 
RUSCELLO, F. (2007). Crisi della famiglia e affidamenti familiari: E1 nuevo art. 155 C.C. Il Diritto di Famiglia e delle Persone, /1, 265-287.

VILLAGRASA ALCAIDE, C. (2012). La custodia compartida en España y en Cataluña: Entre deseos y realidades. Picontó Novales, T. (ed.) La custodia compartida a debate, Madrid, Dykinson-Instituto de Derechos Humanos Bartolomé de las Casas. Universidad Carlos III de Madrid, 77-99. 